\title{
Magnetometry and electron paramagnetic resonance studies of phosphine- and thiol-capped gold nanoparticles
}

\author{
E. Guerrero, ${ }^{1}$ M. A. Muñoz-Márquez, ${ }^{1, a)}$ A. Fernández, ${ }^{1}$ P. Crespo, ${ }^{2}$ A. Hernando, ${ }^{2}$ \\ R. Lucena, ${ }^{3}$ and J. C. Conesa ${ }^{3}$ \\ ${ }_{1}^{1}$ Instituto de Ciencia de Materiales de Sevilla, CSIC-US, Américo Vespucio 49, 41092 Sevilla, Spain \\ ${ }^{2}$ Instituto de Magnetismo Aplicado, UCM-ADIF-CSIC, P.O. Box 155, 28230 Las Rozas, Madrid, Spain and \\ Departamento de Física de Materiales, UCM, 28040 Madrid, Spain \\ ${ }^{3}$ Instituto de Catálisis y Petroleoquímica, CSIC, Marie Curie 2, Campus de Cantoblanco, 28049 Madrid, \\ Spain
}

(Received 9 September 2009; accepted 26 January 2010; published online 16 March 2010)

\begin{abstract}
In the last years, the number of studies performed by wholly independent research groups that confirm the permanent magnetism, first observed in our research lab, for thiol-capped $\mathrm{Au}$ nanoparticles (NPs) has rapidly increased. Throughout the years, the initial magnetometry studies have been completed with element-specific magnetization measurements based on, for example, the $\mathrm{x}$-ray magnetic circular dichroism technique that have allowed the identification of gold as the magnetic moment carrier. In the research work here presented, we have focused our efforts in the evaluation of the magnetic behavior and iron impurities content in the synthesized samples by means of superconducting quantum interference device magnetometry and electron paramagnetic resonance spectrometry, respectively. As a result, hysteresis cycles typical of a ferromagnetic material have been measured from nominally iron-free gold NPs protected with thiol, phosphine, and chlorine ligands. It is also observed that for samples containing both, capped gold NPs and highly diluted iron concentrations, the magnetic behavior of the NPs is not affected by the presence of paramagnetic iron impurities. The hysteresis cycles reported for phosphine-chlorine-capped gold NPs confirm that the magnetic behavior is not exclusively for the metal-thiol system. (C) 2010 American Institute of Physics. [doi:10.1063/1.3327414]
\end{abstract}

\section{INTRODUCTION}

A rather surprising ferromagneticlike behavior has been reported in nanoparticles (hereinafter referred to as NPs) from various transition metals such as $\mathrm{Ag}, \mathrm{Au}$, and $\mathrm{Pd}$ which are nonmagnetic as bulk materials. ${ }^{1-4}$ Among these systems, gold has attracted much of the attention due to its sound potential in biomedical and catalytic applications. ${ }^{5,6}$ Permanent magnetism in thiol-capped gold NPs has already been confirmed by wholly independent research groups throughout the world using different experimental techniques. ${ }^{7-11}$ Some of these works are based on element-specific techniques such as $\mathrm{x}$-ray magnetic circular dichroism (XMCD) on the gold L-edge ${ }^{8,9}$ and, the most recent study by Garitaonandia et al. ${ }^{11}$ has combined XMCD measurements with ${ }^{197} \mathrm{Au}$ Mössbauer spectroscopy to study the magnetic behavior of gold NPs, which are identical to the synthesized thiolcapped NPs studied in the research work presented here. As a result of the research carried out with element-specific techniques, it has been univoquely determined that the gold atoms in the nanoparticle surface are carriers of the magnetic moment.

Furthermore, there have been some experimental studies on the electronic structure of thiol-capped gold NPs ${ }^{12-14}$ that are focused on the potential relationship between the surface plasmon resonance observed for these NPs and the measured

\footnotetext{
${ }^{\text {a) }}$ Author to whom correspondence should be addressed. Electronic mail: miguel.angel@icmse.csic.es.
}

magnetic behavior. The evolution of the ferromagneticlike behavior with the NPs microstructure has also been subject of research ${ }^{15-17}$ and theoretical investigations ${ }^{18-21}$ have recently corroborated the experimental results. All these efforts have provided new insights to discern the origins of the ferromagneticlike behavior in capped transition-metal NPs.

Despite the experimental proofs which strongly conclude that there is a magnetic moment associated to the gold atoms bonded to thiol ligands in the NP surface, the eventual influence of magnetic impurities in part of the magnetic behavior observed in these systems has always been remarked as a key point yet to be addressed, impurities of the chemical reagents used throughout the synthesis process, Fe atoms incorporated from the tools used to manipulate the samples, etc. These are a more than probable source of iron that, in principle, could account for a fraction of the observed magnetic behavior. ${ }^{22}$ On this regard, the role of magnetic iron impurities in the ferromagnetic behavior of gold NPs has been recently subject of research, ${ }^{23}$ where a significant amount $(\sim 1 \mathrm{wt} \%)$ of superparamagnetic $\mathrm{Fe}$ was added to the samples with the aim of determining how this iron could affect the measured hysteresis cycle. Most probably those superparamagnetic impurities did contain traces of iron oxide. However, no investigation has been reported on the influence of diluted paramagnetic iron impurities in the magnetic properties of gold NPs, when the Fe:Au ratio is close or below one iron atom per gold NP, being this the expected situation for eventual incorporation of $\mathrm{Fe}$ impurities during synthesis and manipulation of NPs when using reagent grade chemicals. 
In the research presented here, the magnetic behavior of gold NPs protected with phosphine, thiol and sometimes, a small content of chlorine ligands is investigated using superconducting quantum interference device (SQUID) magnetometry, while the magnetic state of eventual iron impurities has been evaluated by means of electron paramagnetic resonance (EPR) spectroscopy. The morphology and chemical composition of Au NPs have also been studied by means of transmission electron microscopy (TEM), scanning electron microscopy (SEM), and inductively coupled plasma (ICP)atomic emission spectrometry analysis. In addition, energydispersive $\mathrm{x}$-ray analysis (EDX) was performed with both, the TEM and the SEM microscopes. Although it has been demonstrated that aggregates of superparamagnetic Fe impurities weaken the ferromagnetic behavior of $\mathrm{Au} \mathrm{NPs}^{23}$ by reducing the strong local anisotropy field of the gold NPs surface, as previously said, there is no information available on the influence of diluted $\mathrm{Fe}$ impurities in the NPs magnetic behavior. Consequently, samples containing both, gold NPs and dispersed paramagnetic $\mathrm{Fe}^{3+}$ ions have been included in this investigation in order to be compared with nominally iron-free samples only containing Au NPs. It must be noted that all the studied samples contain NPs which have roughly the same size, therefore, size effects can be ruled out from an eventual observation of a magnetic behavior change.

\section{EXPERIMENTAL}

\section{A. NP synthesis}

Triphenylphosphine-stabilized gold NPs, sample labelled as Au-TPP, have been prepared following a synthesis method, ${ }^{24}$ which is a modification of the Brust protocol. ${ }^{25} 0.2$ $\mathrm{g}$ of $\mathrm{HAuCl}_{4}$ (Fluka, 99\%) were dissolved in $12 \mathrm{ml}$ of milli-Q water and then mixed with a solution of $0.28 \mathrm{~g}$ of tetraoctylammonium bromide (Aldrich, 98\%) in $12 \mathrm{ml}$ of toluene which was used as phase-transfer agent. At this point, the $\mathrm{Au}(\mathrm{III})$ is transferred into the organic phase which turns orange and is separated from the aqueous phase. Once the organic phase is separated, it is strongly stirred for five minutes while $0.46 \mathrm{~g}$ of solid triphenylphosphine $\left(\mathrm{PPh}_{3}\right.$, Fluka, 98.5\%) are added. Then the solution is further stirred for ten more minutes until the organic phase was white and cloudy. A fresh solution of $\mathrm{NaBH}_{4} 99 \%$ purchased from Aldrich ( $0.4 \mathrm{~g}$ of solute in $2 \mathrm{ml}$ of milli-Q water) is quickly added while stirring. The organic phase turned dark and was continuously stirred for three more hours under nitrogen flow. The toluene is then removed under reduced pressure by means of a rotary evaporator to yield a black solid, which is then redissolved in hexane and subsequently filtered. The resulting solid was washed with hexane and $\mathrm{MeOH} / \mathrm{H}_{2} \mathrm{O}$ to remove the phase-transfer catalyst, byproducts, and unreacted starting materials. The Au-TPP synthesis would result in approximately $2 \mathrm{~nm}$ NPs with an estimated formula $\mathrm{Au}_{225}\left(\mathrm{PPh}_{3}\right)_{80} \mathrm{Cl}_{16}$. It must be noted that solvents (toluene, hexane, milli-Q water, $\mathrm{MeOH}$, and $i$ - $\mathrm{PrOH}$ ) were dry distilled in our lab each time to be used fresh for every reaction in this and any other synthesis process described in this research work.
Thiolated gold NPs, labelled as Au-SR exchange, $_{\text {, were syn- }}$ thesized via ligand exchange reactions of phosphinestabilized NPs (Au-TPP) as previously described by Woehrle et $\mathrm{al}^{26}{ }^{26}$ The starting point was a solution of $0.156 \mathrm{~g}$ of AuTPP in $40 \mathrm{ml}$ of $\mathrm{CH}_{2} \mathrm{Cl}_{2}$, to this solution $0.156 \mathrm{~g}$ of dodecanethiol (Aldrich, 98.5\%) were added. The resulting mixture was stirred at room temperature for $10 \mathrm{~h}$ to complete the ligand exchange reactions. Afterwards, the solvent is removed under a stream of nitrogen. At this stage, we modified the original synthesis method. The product was dissolved in ethanol and the nonsoluble part was filtered. This filtration process helped us to remove a fractional phase of subnanometric thiol-capped gold clusters. The remaining product contained two phases, thiol-capped metal NPs and a polymerlike compound. This product was gel filtrated over sephadex LH-20 using toluene/ $i-\mathrm{PrOH}$ (concentration ratio of 2:1) as eluent, which eliminated most of the polymericlike component. After the gel filtration procedure, it was found that the resulting sample, which should contain monodisperse thiol-capped gold NPs, actually had a noticeable size dispersion as it can be inferred from the size distribution histograms extracted from the TEM micrographs. NPs sizes between 2 and $2.5 \mathrm{~nm}$ are observed, these diameters are consistent with metallic cores of 180 to 459 gold atoms. As it will be proved later by the chemical composition results, after all the filtration procedures, some phosphine ligands still remain in the sample, which means that the Au-TPP NPs have not undergone a full exchange reaction and/or there are some fractional polymeric-like phases left, e.g., $\mathrm{PPh}_{3}-\mathrm{Au}-\mathrm{SR}$, which were not completely removed by the thorough filtration stages. However, the incomplete exchange reaction hypothesis is more than plausible since the chemical synthesis of capped gold NPs in organic solutions is keen to induce competition between the thiol and phosphine ligands as it has been reported before. ${ }^{27}$

Finally, two batches of thiolated gold NPs were prepared following the synthesis method developed by Brust et al., ${ }^{25}$ which authors have used elsewhere. ${ }^{16}$ The first set resulted in thiol-capped Au NPs containing some paramagnetic dispersed $\mathrm{Fe}^{3+}$, related with the purity of the thiols used in the synthesis process (Aldrich 98\%). These NPs are named $\alpha-\mathrm{Au}-\mathrm{SR}_{\mathrm{Brust}}$. The second set was synthesized using dodecanethiol (Aldrich 98.5\%) with a highest degree of purity and, as it will be discussed later, it was found to be free from any iron impurity as determined by EPR, chemical analysis, and EDX, this sample was labelled as $\beta$-Au-SR $\mathrm{Brust}_{\text {. }}$

\section{B. Characterization techniques}

The iron and gold content in the synthesized samples was evaluated by ICP-atomic emission spectrometry analysis using a Fisions ARL-3410 ICP kit. The total amount of light elements in the samples, such as carbon, hydrogen, phosphorus, and sulphur, was determined by elemental chemical analysis with a Leco CHNS 93. These techniques will provide a chemical composition estimation of the whole sample, therefore, bonded and unbonded ligand molecules are measured. Instead, the EDX analysis at the electron microscope allows the chemical composition determination in a well- 
TABLE I. Average size and chemical composition (weight percentage, wt \%) data for the selected samples. The Fe content in all the studied samples has been found to be below the ICP sensitivity which in this case is 0.03 wt $\%$.

\begin{tabular}{|c|c|c|c|c|c|c|c|c|}
\hline Sample & $\mathrm{Au}$ & $\mathrm{S}$ & $\begin{array}{c}\mathrm{P} \\
\text { wt } \%\end{array}$ & $\mathrm{C}$ & $\mathrm{H}$ & $\begin{array}{l}\text { Au:ligand }{ }^{\mathrm{a}} \\
\text { (at. ratio) }\end{array}$ & $\begin{array}{l}\mathrm{Dm}^{\mathrm{b}} \\
(\mathrm{nm})\end{array}$ & $\begin{array}{c}\sigma^{\mathrm{c}} \\
(\mathrm{nm})\end{array}$ \\
\hline $\mathrm{Au}-\mathrm{TPP}$ & 43.5 & $\ldots$ & 5.9 & 41.1 & 2.9 & 3.3 & 2.1 & 0.2 \\
\hline \multirow{2}{*}{ Au-SR exchange } & 24.2 & 1.5 & 7.0 & 55.6 & 5.0 & 2.5 & 1.87 & 0.14 \\
\hline & & & & & & & 2.84 & 0.18 \\
\hline$\alpha$-Au-SR ${ }_{\text {Brust }}$ & 50.3 & 4.1 & $\ldots$ & 24.3 & 4.4 & 2.0 & 2.1 & 0.2 \\
\hline$\beta$-Au-SR & 51.5 & 6.8 & $\cdots$ & 30.4 & 5.5 & 1.2 & 2.23 & 0.15 \\
\hline
\end{tabular}

a $\mathrm{Au}: \mathrm{P}$ atomic ratio for the Au-TPP sample and Au:S ratio for all the other samples determined by TEM-EDX.

${ }^{\mathrm{b}}$ Average particle size as calculated from particle size distribution histograms.

${ }^{\mathrm{c}}$ Standard deviation of the log-normal functions used in the histogram fit which corresponds to the size distribution.

defined and small region of the sample, which will provide a better approximation to the actual gold to ligand ratio. EDX was performed at the Philips CM200 TEM microscope, however, due to the lower cross section of $200 \mathrm{keV}$ electrons to light elements, the presence of chlorine atoms in the Au-TPP sample had to be checked with a Hitachi S-4800 SEM microscope at $20 \mathrm{keV}$, the electrons have a higher cross section so chlorine could be detected.

The ICP and chemical analysis results that provide information on the chemical composition of the studied samples along with the NPs diameters and the gold to ligand ratio measured with TEM are summarized in Table I. There are three points that should be remarked here. The first one, concerns the precision of the results presented in Table I, the elemental composition should add upto $100 \mathrm{wt} \%$, however, this is not the case and could be attributed to a variation in the instrument response and machine drift as a function of mass. Drift and response are nonlinear and, the degree of deviation differs from one mass to another. ${ }^{28}$ Additionally, digestion of samples might have not been complete leading to a slight underestimation of the composition. Secondly, the chemical analysis of sample Au-SR exchange indicates the presence of phosphorus in the sample inspite of the purification process followed. This indicates, as already mentioned, that the Au-TPP NPs have not undergone a full exchange reaction and/or there are some phosphine-containing phases remaining in the sample. Finally, the iron content was always below the detection limits of the ICP technique which, in this case, is $0.03 \mathrm{wt} \%$. This means that a more sensitive technique was needed in order to determine whether the samples contained traces of iron below the aforementioned detection limits. Since EPR is a spectroscopic technique that detects chemical species that have unpaired electrons then, it was proved to be a well-suited experimental technique to detect traces of iron, in addition, the EPR spectrum can provide information regarding the magnetic state of the detected element as it will be discussed later.

Regarding the magnetism experiments, the SQUID measurements were performed using a Quantum Design SQUID magnetometer. Hysteresis cycles were recorded for every single sample at 5 and $300 \mathrm{~K}$. The used sample holder was adhesive kapton stuck to a quartz tube. The diamagnetic contribution from the sample holder was measured and subtracted from the total magnetization. Meanwhile, the EPR measurements were carried out in a Bruker ER200D instrument operating in the $\mathrm{x}$-band and interfaced to a digital data acquisition system. Aliquots of the studied samples were placed into a special spectroscopically pure quartz cell. All the spectra were recorded in a $\mathrm{TE}_{104}$-type double cavity. The frequency of the microwave was calibrated for each experiment using a standard of the stable free radical diphenylpicrylhydrazyl located in the second cavity, which has a Landé $\mathrm{g}$-factor $\mathrm{g}_{e}=2.0036$.

\section{RESULTS AND DISCUSSION}

The TEM micrographs and subsequent size distribution analysis are shown in Fig. 1. The size distribution histograms representative of each sample have been fitted using a lognormal function, which gives the NP mean size and the size dispersion, this function often reproduces the NP size distribution. ${ }^{29,30}$ Log-normal functions describe very well the size distribution of closed systems, i.e., constant mass, where aggregation is the main growth mechanism. In terms of monodispersion, these fits point out the good quality of the samples Au-TPP, $\alpha$-Au-SR Brust $_{\text {, }}$ and $\beta$-Au-SR Brust . Regarding the Au-SR exchange $_{\text {sample, a significant broadening of size }}$ dispersion is observed, this could have been originated by the exchange reaction that takes place in this case. As it has been described before, ${ }^{31}$ in these exchange reactions part of the ligand shell is replaced as a metal-ligand entity, i.e., $\mathrm{AuCl}\left(\mathrm{PPh}_{3}\right)$, leading to loss of metal core atoms (etching process). This would lead to the production of approximately $1.9 \mathrm{~nm}$ particles during the ligand exchange reaction. Additionally, since the exchange process is prone to be rather complex, comparing competition between thiol and phosphine ligands, ${ }^{27}$ once the NP core has lost some of the phosphine ligands attached to gold core atoms then, the NP core is exposed to the liquid phase, which would allow further aggregation of metallic dispersed gold leading to an increase in the NP size (approximately $2.8 \mathrm{~nm}$ particles). Hence, a bimodal log-normal function will describe very accurately the experimental results. Finally, the marginal difference $(\sim 0.1 \mathrm{~nm})$ between the $\alpha$-Au-SR Brust $_{\text {and }} \beta$-Au-SR Brust $_{\text {NPs }}$ is not significant since slight variations in the synthesis conditions along with the experimental error of the size determination process may lead to this size difference. 

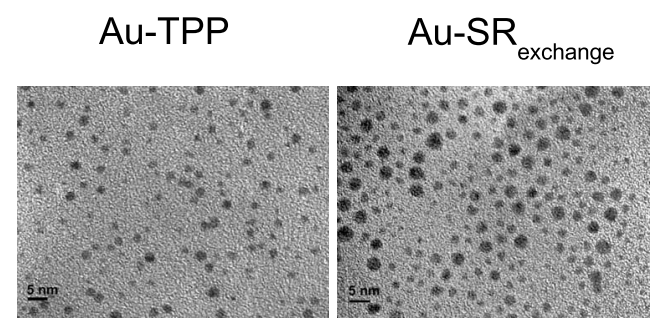

$\mathrm{Dm}=2.1 \mathrm{~nm}$ $\sigma=0.2 \mathrm{~nm}$

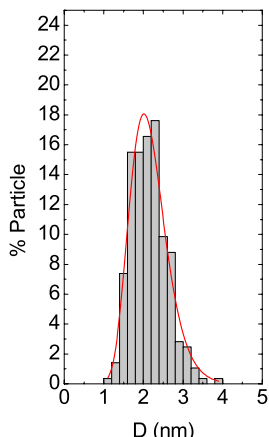

$\mathrm{Dm}_{1}=1.87 \mathrm{~nm} ; \mathrm{Dm}_{2}=2.84 \mathrm{~nm}$

$\sigma_{1}=0.14 \mathrm{~nm} ; \sigma_{2}=0.16 \mathrm{~nm}$

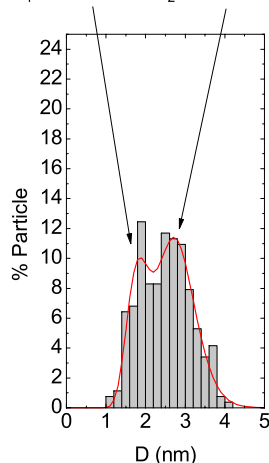

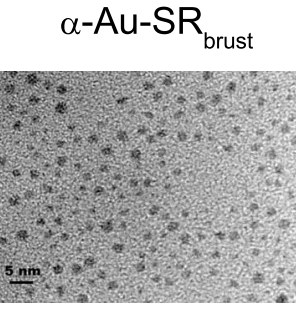

$\mathrm{Dm}=2.1 \mathrm{~nm}$

$\sigma=0.2 \mathrm{~nm}$

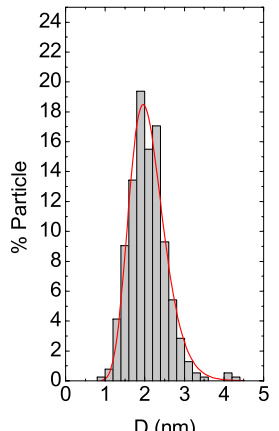

$\beta-A u-S R_{\text {brust }}$
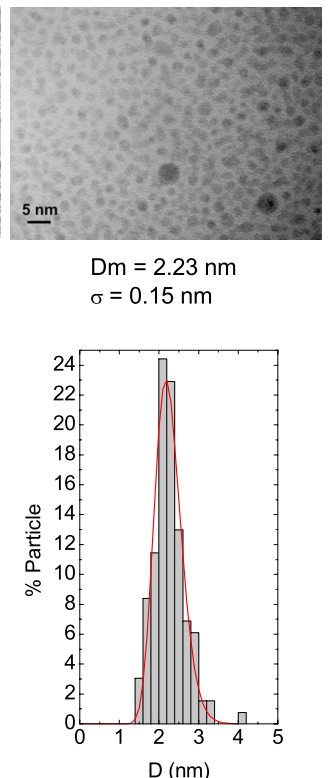

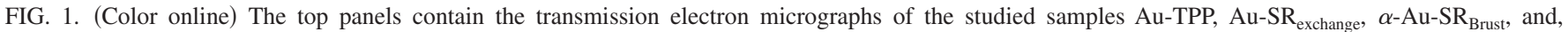

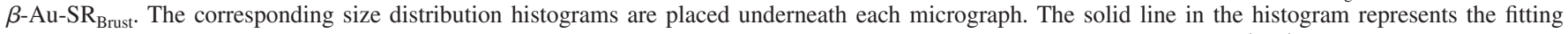
curve assuming that the size distribution is well reproduced by a log-normal function. The calculated mean particle diameter (Dm) and the standard deviation $(\sigma)$ are included in the histograms. It must be noted that the size distribution of the Au-SR exchange sample is noticeably broader than the rest. Therefore, this histogram is best fitted with a bimodal log-normal function.

The SQUID analysis of the Au-TPP sample is presented in Fig. 2(a), at 5 and $300 \mathrm{~K}$. In this sample, the magnetic saturation remains almost constant throughout the temperature range and only the coercive field decreases from 850 down to 300 Oe when raising the temperature from 5 to 300 $\mathrm{K}$. The magnetic behavior of the Au-SR $\mathrm{R}_{\text {exchange }}$ sample, shown in Fig. 2(b) at 5 and $300 \mathrm{~K}$, presents two clear contributions, a paramagnetic component and a ferromagneticlike component very similar to the analogous contribution observed in the Au-TPP sample, as inferred from the magnetization curve obtained at $300 \mathrm{~K}$. The paramagnetic component becomes evident in the high field range of the low temperature hysteresis loop curve. This component almost disappears at high temperature as predicted by the Curie law. Finally, Figs. 2(c) and 2(d) show the hysteresis cycles of the

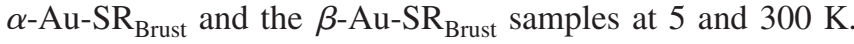
As discussed before, there is a difference in these two samples, the $\alpha$-Au-SR $\mathrm{Brust}_{\text {Brt }}$ sample contains a non-negligible amount of dispersed paramagnetic $\mathrm{Fe}^{3+}$ impurities as it will be discussed later once the EPR measurements are presented. However, both samples exhibit at low temperature a neat paramagnetic contribution, similarly to sample $\mathrm{Au}-\mathrm{SR}_{\text {exchange. }}$ According to the EPR analysis, the paramagnetism of samples Au-SR exchange and $\beta$-Au-SR $\mathrm{Brust}_{\text {cannot be }}$ originated by $\mathrm{Fe}$ impurities, therefore, $\mathrm{Au}$ atoms with nonblocked magnetic moment could be in the origin of this contribution. Another possibility would be the presence of a fraction of thiol-proctected $\mathrm{Au}_{25}$ atom clusters, which have been shown to perform as a paramagnetic system under certain conditions, ${ }^{32}$ however, the size distribution histograms do not show a significant fraction of these clusters that could account for such an important paramagnetic signal.
However, despite differences in the iron content and paramagnetic contributions, the measurement of the hysteresis loop at $300 \mathrm{~K}$ indicates that for all the studied samples there is no difference at all in their respective ferromagnetic behaviors other than a minor variation in the coercive field, which is slightly lower in the iron containing sample $\alpha$-Au-SR $\mathrm{Brust}_{\text {at }}$ at both high and low temperature.

The magnetization results obtained from the SQUID experiments in our samples at $300 \mathrm{~K}$ are summarized in Table II. The measured magnetization values for the ferromagnetic component range from $0.02-0.24 \mu_{\mathrm{B}} / \mathrm{NP}$. These values are comparable with the magnetization values associated to the Au $5 d$ orbitals as measured with XMCD experiments by Garitaonandia et al. ${ }^{11}$ and, more recently, by de la Venta et $a l .{ }^{33}$ A typical value in the range of $0.002 \mu_{\mathrm{B}} / \mathrm{Au}$ atom has been obtained. If we take into consideration that only the surface gold atoms have the localized charge transfer, the typical magnetization values that we measure can be increased by a factor of 1.5 to 2 (for NPs with a core diameter ranging from 1.9-2.8 nm). Consequently, we found fractional magnetization values below $1 \mu_{\mathrm{B}}$, which confirm that the electron is partially shared between the surface metal atom and the organic ligand. This fact is reflected in the $5 d$ hole generation due to different bonding between the surface metal atoms and the organic ligand. Finally, it is worth remarking that blocking behavior exists for magnetization values well below one $\mu_{\mathrm{B}}$ since the blocking has its origin in the spin-orbit field. ${ }^{34}$

EPR experiments were carried out at room temperature $(298 \mathrm{~K})$ and also at liquid nitrogen temperature $(77 \mathrm{~K})$ for all the studied samples, Au-TPP, Au-SR exchange, $\alpha$-Au-SR $\mathrm{Brust}_{\text {, }}$

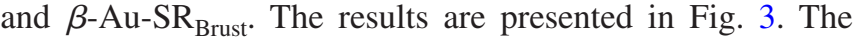




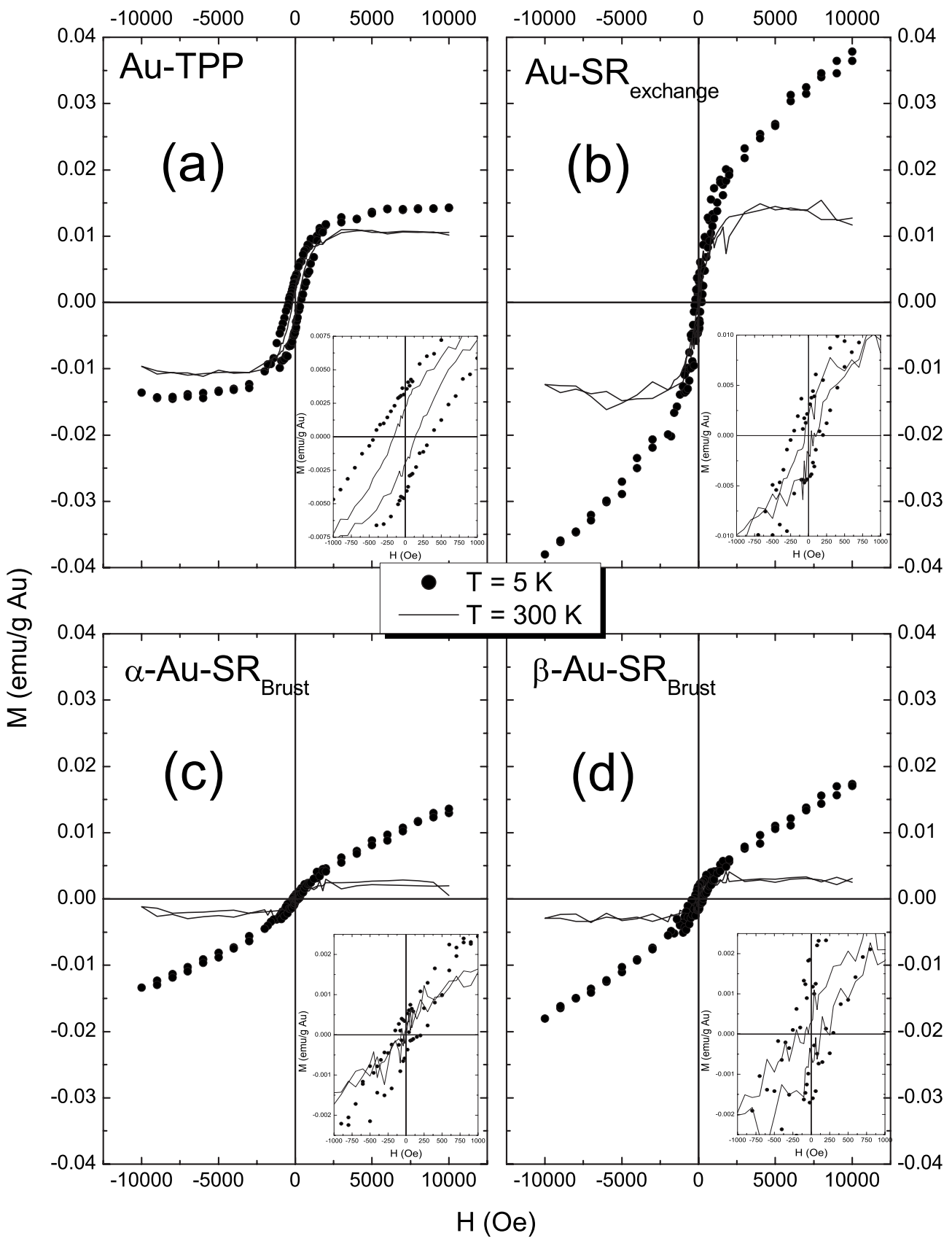

FIG. 2. SQUID measurements from all the studied samples, top panels correspond to Au-TPP (a) Au-SR exchange $(\mathrm{b}), \alpha$-Au-SR $\mathrm{Brust}_{\text {(c) }}$ (c), and $\beta$-Au-SR $\mathrm{Brust}$ (d) are presented in the bottom panels. Spectra were measured at $\mathrm{T}=5 \mathrm{~K}$ and $\mathrm{T}=300 \mathrm{~K}$.

TABLE II. Magnetic saturation for the studied samples. The values in $\mathrm{emu} / \mathrm{g}_{\mathrm{Au}}$ were obtained from the experimental hysteresis curves at $300 \mathrm{~K}$ along with the gold concentration determined from the ICP results. The magnetization per NP has been calculated by estimating the number of gold atoms in the NP core, as previously described (Refs. 38 and 39).

\begin{tabular}{|c|c|c|c|}
\hline Sample & $\mathrm{Au}$ atoms/NP & $\begin{array}{c}\mathrm{M}_{\mathrm{S}} \\
\left(\mathrm{emu} / \mathrm{g}_{\mathrm{Au}}\right)\end{array}$ & $\begin{array}{c}\mathrm{M}_{\mathrm{S}} \\
\left(\mu_{\mathrm{B}} / \mathrm{NP}\right)\end{array}$ \\
\hline Au-TPP & 225 & 0.010 & 0.08 \\
\hline Au-SR exchange & $180-459$ & 0.015 & $0.10-0.24$ \\
\hline$\alpha$-Au-SR $\mathrm{Brust}_{\text {Brt }}$ & 180 & 0.003 & 0.02 \\
\hline$\beta$-Au-SR Brust & 225 & 0.004 & 0.03 \\
\hline
\end{tabular}

most significant feature is found in the EPR spectra of the

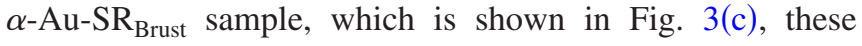
spectra present a peak around 1600 Gauss $\left(\mathrm{g}_{e}=4.28\right.$ at crossover point) typical of dispersed high spin $\mathrm{Fe}^{3+} \cdot 35$ The intensity of this peak is lower (peak-to-peak amplitude approximately five times smaller) at $298 \mathrm{~K}$, which would be in agreement with the typical signal behavior of a paramagnetic centre, the EPR signal decreases as the temperature increases. Likewise, a somewhat broader feature is observed in the low temperature EPR spectrum of the $\beta$-Au-SR $\mathrm{Brust}_{\text {int }}$ sample, which also vanishes at high temperature. This could be attributed to a highly diluted concentration of paramagnetic $\mathrm{Fe}^{3+}$, close to the detection limits of the technique. However, as it will be discussed now, this feature at approxi- 


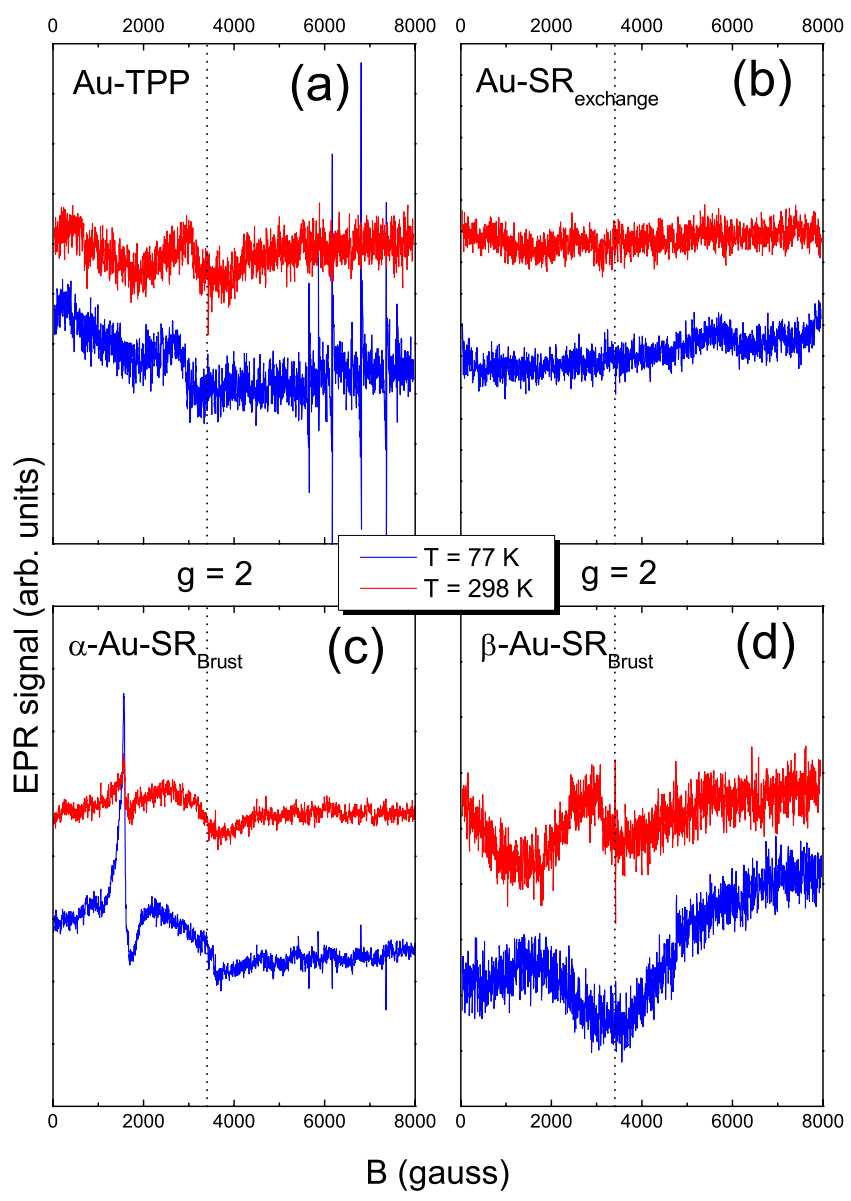

FIG. 3. (Color online) EPR spectra from all studied samples, Au-TPP panel

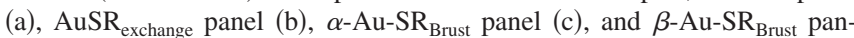
el(d). Data were collected at $\mathrm{T}=298 \mathrm{~K}$ and $\mathrm{T}=77 \mathrm{~K}$. A fiducial line represents the free electron g-factor.

mately 1600 Gauss in the EPR spectrum at $77 \mathrm{~K}$ of the

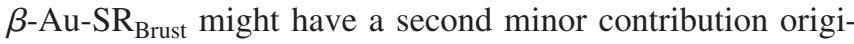
nated by a ferromagnetic centre.

In fact, a broad resonance peak can be found at low $\mathrm{g}$ values $(\sim 3000 \mathrm{Oe})$, which at low temperatures increases its linewidth and shifts to higher $g$ values. This feature can be seen in almost all the EPR spectra with more or less intensity. In the EPR spectrum of the Au-TPP sample is quite distinct while it is really shallow in the high temperature curve of sample Au-SR exchange and completely negligible in the low temperature spectrum of the same sample. Regarding samples $\alpha$-Au-SR $\mathrm{SR}_{\mathrm{Brust}}$ and $\beta$-Au-SR $\mathrm{SR}_{\mathrm{Brust}}$, this resonance at approximately 3000 Oe is overlapped in the low temperature curve with the resonance peak of the $\mathrm{Fe}^{3+}$ centres, specially

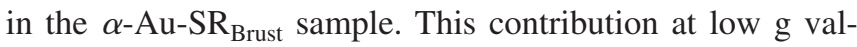
ues, which broadens at low temperature and also shifts to lower magnetic field might be attributed to ferromagnetic centres $^{36,37}$ such as our capped gold NPs.

Finally, one should note that, in the EPR spectrum of the Au-TPP sample, four spiky peaks appear between 5500 and 7500 Gauss, which correspond to paramagnetic $\mathrm{O}_{2}$ molecules present in the analysis chamber due to slightly higher pressure conditions in this specific case.

The results obtained here from the combined SQUID and EPR study are complementary to previous investigations regarding the effect of iron impurities on the magnetic behavior of $\mathrm{Au} \mathrm{NPs}^{23}$ where a significant amount $(\sim 1$ wt $\%)$ of iron impurities were added. The current research is focused on the effects produced by highly diluted iron concentrations, which are in the range of one iron atom per NP. As a result, the magnetic behavior is either confirmed for iron-free phosphine- and thiol-stabilized gold NPs and also for thiol-capped gold NPs containing dispersed paramagnetic $\mathrm{Fe}^{3+}$ impurities. In addition, the hysteresis cycle measured in iron-free samples reinforces the previous experimental works that claimed ferromagneticlike behavior in systems that are diamagnetic as bulklike materials. ${ }^{7-9}$ Moreover, the ferromagneticlike behavior is verified for gold NPs protected with phosphine ligands.

\section{CONCLUSIONS}

In conclusion, the strong ferromagneticlike character has been corroborated by means of SQUID magnetometry for phosphine-chlorine-capped gold NPs, this includes the Au$\mathrm{P} / \mathrm{Au}-\mathrm{Cl}$ bonds in the set of capping systems suitable for inducing ferromagnetic behavior in gold NPs. Since the phosphine-capped NPs do not contain iron impurities that could alter the hysteresis cycle, another proof is provided for concluding that ferromagnetism of gold NPs is not originated by magnetic impurities, which agrees with the previous XMCD and Mössbauer spectroscopy results. Thiol-capped gold NPs samples obtained following different synthesis routes with or without dispersed paramagnetic $\mathrm{Fe}^{3+}$ impurities also show a ferromagneticlike behavior. These thiolcapped samples show a paramagnetic component, which most probably is originated by gold atoms with a nonblocked magnetic moment. Regarding the NPs obtained following the Brust method, despite a slight difference detected in the coercive field measured for the $\alpha$-Au-SR $\mathrm{Brust}_{\text {and }} \beta-\mathrm{Au}-\mathrm{SR}_{\mathrm{Brust}}$ samples, which is difficult to confirm due to the noise:signal ratio, it has been found that dispersed paramagnetic $\mathrm{Fe}^{3+}$ impurities do not affect the magnetic behavior observed.

Finally, the EPR experiments confirm the $\mathrm{Fe}^{3+}$ content in

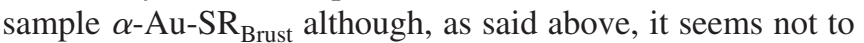
have any effect on the observed magnetism. The EPR spectra also show a faint peak at low g values in all the samples (being very weak in the Au-SR exchange sample), which could be attributed to the ferromagnetic capped gold NPs.

\section{ACKNOWLEDGMENTS}

This research has been supported by the Spanish MECINN (Project Consolider-Ingenio in Molecular Nanoscience, CSD2007-00010) and "Junta de Andalucía" (Project Nos. P06-FQM-02254 and P09-FQM-4554, group TEP217). EG thanks the Spanish MEC for financial support and MAMM acknowledges the CSIC I3P program. R. L. thanks CSIC for a PhD grant.

${ }^{1}$ H. Hori, T. Teranishi, Y. Nakae, Y. Seino, M. Miyake, and S. Yamada, Phys. Lett. A 263, 406 (1999).

${ }^{2}$ T. Shinohara, T. Sato, and T. Taniyama, Phys. Rev. Lett. 91, 197201 (2003).

${ }^{3}$ B. Sampedro, P. Crespo, A. Hernando, R. Litrán, J. C. Sánchez-López, C. López-Cartes, A. Fernández, J. Ramírez, J. G. Calbet, and M. Vallet, Phys. 
Rev. Lett. 91, 237203 (2003).

${ }^{4}$ L. Suber, D. Fiorani, G. Scavia, P. Imperatori, and W. R. Plunkett, Chem. Mater. 19, 1509 (2007).

${ }^{5}$ M. P. Melancon, W. Lu, and C. Li, MRS Bull. 34, 415 (2009).

${ }^{6}$ M. Turner, V. B. Golovko, O. P. H. Vaughan, P. Abdulkin, A. BerenguerMurcia, M. S. Tikhov, B. F. G. Johnson, and R. M. Lambert, Nature (London) 454, 981 (2008).

7P. Crespo, R. Litrán, T. C. Rojas, M. Multigner, J. M. de la Fuente, J. C Sánchez-López, M. A. García, A. Hernando, S. Penadés, and A. Fernández, Phys. Rev. Lett. 93, 087204 (2004)

${ }^{8}$ Y. Yamamoto, T. Miura, M. Suzuki, N. Kawamura, H. Miyagawa, T. Nakamura, K. Kobayashi, T. Teranishi, and H. Hori, Phys. Rev. Lett. 93, 116801 (2004).

${ }^{9}$ Y. Negishi, H. Tsunoyama, M. Suzuki, N. Kawamura, M. M. Matsushita, K. Maruyama, T. Sugawara, T. Yokoyama, and T. Tsukuda, J. Am. Chem. Soc. 128, 12034 (2006).

${ }^{10}$ P. Dutta, S. Dal, S. Seehra, M. Anand, and C. B. Roberts, Appl. Phys. Lett. 90, 213102 (2007).

${ }^{11}$ J. S. Garitaonandia, M. Insausti, E. Goikolea, M. Suzuki, J. D. Cashion, N. Kawamura, H. Ohsawa, I. G. de Muro, K. Suzuki, F. Plazaola, and T. Rojo, Nano Lett. 8, 661 (2008).

${ }^{12}$ P. Zhang and T. K. Sham, Phys. Rev. Lett. 90, 245502 (2003).

${ }^{13}$ J. de la Venta, A. Pucci, E. Fernández-Pinel, M. A. García, C. de Julián Fernández, P. Crespo, P. Mazzoldi, G. Ruggeri, and A. Hernando, Adv. Mater. 19, 875 (2007).

${ }^{14}$ E. Guerrero, M. A. Muñoz-Márquez, M. A. García, P. Crespo, E. Fernández-Pinel, A. Hernando, and A. Fernández, Nanotechnology 19, 175701(6) (2008)

${ }^{15}$ Y. Yamamoto and H. Hori, Rev. Adv. Mater. Sci. 12, 23 (2006).

${ }^{16}$ E. Guerrero, T. C. Rojas, M. Multigner, P. Crespo, M. A. Muñoz-Márquez, M. A. García, A. Hernando, and A. Fernández, Acta Mater. 55, 1723 (2007).

${ }^{17}$ E. Guerrero, M. A. Muñoz-Márquez, E. Fernández-Pinel, P. Crespo, A Hernando, and A. Fernández, J. Nanopart. Res. 10, 179 (2008).

${ }^{18}$ W. Luo, S. J. Pennycook, and S. T. Pantelides, Nano Lett. 7, 3134 (2007).

${ }^{19}$ C. Gonzalez, Y. Simón-Manso, M. Marquez, and V. Mujica, J. Phys. Chem. B 110, 687 (2006).

${ }^{20}$ F. Michael, C. Gonzalez, V. Mujica, M. Marquez, and M. A. Ratner, Phys. Rev. B 76, 224409 (2007).

${ }^{21}$ M. Walter, J. Akola, O. Lopez-Acevedo, P. D. Jadzinsky, G. Calero, C. J.
Ackerson, R. L. Whetten, H. Grönbeck, and H. Häkkinen, Proc. Natl. Acad. Sci. U.S.A. 105, 9157 (2008).

${ }^{22}$ D. W. Abraham, M. M. Frank, and S. Guha, Appl. Phys. Lett. 87, 252502 (2005).

${ }^{23}$ P. Crespo, M. A. García, E. Fernández-Pinel, M. Multigner, D. Alcántara, J. M. de la Fuente, S. Penadés, and A. Hernando, Phys. Rev. Lett. 97, 177203 (2006)

${ }^{24}$ W. W. Weare, S. M. Reed, M. G. Warner, and J. E. Hutchison, J. Am. Chem. Soc. 122, 12890 (2000).

${ }^{25}$ M. Brust, M. Walker, D. Bethell, D. J. Schiffrin, and R. Whyman, J. Chem. Soc., Chem. Commun. 7, 801 (1994).

${ }^{26}$ G. H. Woehrle, L. O. Brown, and J. E. Hutchison, J. Am. Chem. Soc. 127, 2172 (2005).

${ }^{27}$ E. E. Foos, M. E. Twigg, A. W. Snow, and M. G. Ancona, Science 19, 573 (2008).

${ }^{28}$ M. M. Cheatham, W. F. Sangrey, and W. M. White Spectrochim. Acta, Part B 48, 487 (1993).

${ }^{29}$ C. G. Granqvist and R. A. Buhrman, J. Appl. Phys. 47, 2200 (1976).

${ }^{30}$ L. B. Kiss, J. Söderlund, G. A. Niklasson, and C. G. Granqvist, Nanotechnology 10, 25 (1999).

${ }^{31}$ G. H. Woehrle and J. E. Hutchison, Inorg. Chem. 44, 6149 (2005).

${ }^{32}$ M. Zhu, C. Aikens, M. Hendrich, R. Gupta, H. Qian, G. Schatz, and R. Jin, J. Am. Chem. Soc. 131, 2490 (2009).

${ }^{33}$ J. de la Venta, V. Bouzas, A. Pucci, M. A. Laguna-Marco, D. Haskel, S. G. E. te Velthuis, A. Hoffmann, J. Lal, M. Bleuel, G. Ruggeri, C. de Julián Fernández, and M. A. Garcí, J. Nanosci. Nanotechnol. 9, 6434 (2009).

${ }^{34}$ A. Hernando, P. Crespo, M. A. García, E. Fernández-Pinel, J. de la Venta, A. Fernández, and S. Penadés, Phys. Rev. B 74, 052403 (2006).

${ }^{35}$ M. Jiang, J. Terra, A. M. Rossi, M. A. Morales, E. M. Baggio Saitovitch, and D. E. Ellis, Phys. Rev. B 66, 224107 (2002).

${ }^{36}$ A. L. Tronconi, P. C. Morais, F. Pelegrini, and F. A. Tourinho, J. Magn. Magn. Mater. 122, 90 (1993).

${ }^{37}$ M. Fittipaldi, L. Sorace, A.-L. Barra, C. Sangregorio, R. Sessoli, and D. Gatteschi, Phys. Chem. Chem. Phys. 11, 6555 (2009).

${ }^{38}$ R. L. Whetten, J. T. Khoury, M. M. Alvarez, S. Murthy, I. Vezmar, Z. L. Wang, P. W. Stephens, C. L. Cleveland, W. D. Luedtke, and U. Landman, Adv. Mater. 8, 428 (1996)

${ }^{39}$ C. López-Cartes, T. C. Rojas, R. Litrán, D. Martínez-Martínez, J. M. de la Fuente, S. Penadés, and A. Fernández, J. Phys. Chem. B 109, 8761 (2005). 